(e-migrinter

e-Migrinter

$12 \mid 2014$

Les chiffres de l'immigration : un regard critique sur les statistiques

\title{
Migrations internationales et santé mentale : enquêter dans les services psychiatriques au Sénégal
}

Séminaire d'équipe Migrinter 2013-2014 : «Quelles sources et méthodes pour la recherche sur les migrations?»

\section{Anne-Laure Counilh}

\section{OpenEdition}

\section{Journals}

Édition électronique

URL : https://journals.openedition.org/e-migrinter/358

DOI : 10.4000/e-migrinter.358

ISSN : 1961-9685

Éditeur

UMR 7301 - Migrinter

Édition imprimée

Date de publication : 15 octobre 2014

Pagination : $99-102$

ISSN : 1961-9685

Référence électronique

Anne-Laure Counilh, " Migrations internationales et santé mentale : enquêter dans les services

psychiatriques au Sénégal », e-Migrinter [En ligne], 12 | 2014, mis en ligne le, consulté le 20 mai 2021

URL : http://journals.openedition.org/e-migrinter/358 ; DOI : https://doi.org/10.4000/e-migrinter.358 


\title{
Séminaire d'équipe Migrinter 2013-2014 : «Quelles sources et méthodes pour la recherche sur les migrations? »
}

\section{Second cycle, séance par Véronique Petit le jeudi 20 mars 2014 : «Migrations internationales et santé mentale : enquêter dans les services psychiatriques au Sénégal »}

\author{
Compte rendu par Anne-Laure Counilh
}

$\mathbf{L}$

e 20 mars 2014 s'est tenue la deuxième séance du $2^{\text {ème }}$ cycle du séminaire « Sources et méthodes » organisé par Kamel Doraï, Marie-Antoinette Hily et David Lessault. L'objectif du cycle de séminaires « Sources et méthodes " est d'approfondir la réflexion menée sur les différentes sources et méthodes utilisées dans les recherches sur les migrations, notamment en s'écartant des sources et méthodes habituelles.

L'entreprise vise à penser autrement les migrations en plaçant celles-ci en tant qu'objet au cour des questionnements.
La séance proposée par Véronique Petit se présente comme le retour d'une expérience de recherche menée dans des services psychiatriques au Sénégal avec Mouhamed Ly et Guilia Pizzolato au sein de l'ANR Miprimo ${ }^{1}$. L'équipe entend analyser la production et la circulation des récits sur la migration en Afrique de l'Ouest. Véronique Petit a pour sa part présenté la façon dont cette parole particulière a été recueillie et son intérêt dans une perspective d'analyse des récits. Elle s'attache à montrer en quoi une parole traditionnellement considérée comme marginale, voire peu fiable, peut se révéler pertinente et novatrice dans l'observation des mobilités.

Dans le cadre de cette recherche, plusieurs partis pris s'imposent. Celui de ne pas isoler les migrants comme objet de recherche mais d'analyser leur récit parmi ceux d'autres personnes considérées comme non migrantes permet de ne pas construire de catégorie a priori. De la même façon, l'objectif de cette recherche est d'élargir les matériaux de recherche afin de pouvoir enrichir la réflexion par l'analyse de différents types de récits: aux méthodes narratives classiques s'ajoute l'étude de productions artistiques et littéraires. Ce choix résulte d'une volonté forte de ne pas aseptiser l'objet de recherche en ne se focalisant que sur un seul mode de recueil de l'information. Ce type de méthode met en

\footnotetext{
${ }^{1}$ ANR 2011-2013 : durant cette période, plusieurs séjours ont été effectués sur le terrain au Sénégal à Thiès, Dakar, Thiaroye, St Louis.
} 
avant la porosité entre les différentes sphères sociales de production des représentations.

Au sein du projet Miprimo, Véronique Petit a souhaité s'intéresser plus particulièrement à la santé mentale à la fois pour des raisons personnelles et suite à l'influence de certaines rencontres lors de ses précédentes expériences sur le terrain. Elle s'interroge alors sur les différences de traitement face à la santé mentale et se replonge dans Histoire de la folie à l'âge classique $^{2}$. Également influencée par les travaux de Marcel Gauchet, sa vision de l'asile (et des services psychiatriques) s'élargit et passe du seul lieu d'enfermement et de répression à un lieu à fonction thérapeutique mais surtout à un lieu qui reflète les changements sociaux et politiques à l'œuvre dans la société. Cette recherche naît à la fois de la constatation d'un manque, celui de l'absence d'attention portée à la psychologie et à la psychiatrie dans les recherches en Afrique (comme le fait remarquer Gérard Salem dans son étude sur la dépression au Sénégal), mais aussi d'un questionnement sur le récit de ceux qui sont considérés comme «fous » ou « inadaptés » par leur société. À l'image des travaux de John et Jean Comaroff, cette étude réhabilite la parole de ces malades et articule sa réflexion autour de la lucidité de ces récits. Cette recherche s'attache donc à considérer la santé mentale comme un indicateur du changement social à différents niveaux (famille, communauté, État). Cette logique s'inscrit dans la continuité des travaux visant à interroger le migrant comme révélateur du lien social à travers sa position dans la société. Ce type d'étude permet d'approfondir la réflexion sur les processus d'individuation et d'individualisation.

Véronique Petit a tenu à expliquer de manière approfondie son choix de s'intéresser à la parole de personnes pensionnaires de services psychiatriques au Sénégal. L'histoire particulière de

${ }^{2}$ L'ouvrage issu de la thèse de doctorat d'État de Michel Foucault. l'implantation de la psychiatrie dans ce pays permet d'étayer les aspects méthodologiques de la recherche. Plusieurs facteurs contextuels permettent de préciser ces choix. Tout d'abord, la société sénégalaise est une société complexe où la prise de parole est régulée et hiérarchisée en fonction des appartenances et de la position sociale. Dans ce cadre, la production des récits est donc très codifiée. Le contexte spécifique des établissements de santé mentale confère à la parole une valeur thérapeutique. Si dans le contexte des établissements psychiatriques, cette parole est alors libérée des contraintes sociales tacites qui pèsent sur la production des discours, elle est souvent déconsidérée. La représentation sociale de la folie au Sénégal est liée à une forte stigmatisation, une violente répression, un rejet de la famille et une volonté d'invisibilisation des malades. L'intérêt de produire une anthropologie de la parole des malades permet de construire un récit différent de celui issu des études classiques des migrations en étudiant les liens entre folie et migrations et en s'intéressant aux aspects pathogènes de la migration $^{3}$. L'histoire de la psychiatrie au Sénégal est particulière: depuis l'indépendance, la fragilité mentale est corrélée à l'urbanisation et à la mobilité. La mobilité des individus est souvent considérée comme facteur déstabilisateur et élément de rupture fort dans l'histoire de vie des migrants. Pourtant, du point de vue des malades et des familles, l'association entre migration et santé mentale est moins évidente. En effet, au sein des familles sénégalaises, la migration est souvent considérée comme s'inscrivant dans la continuité des pratiques traditionnelles et constitue une expérience valorisée par les familles et la société. Ce discours est antinomique avec celui des médecins, mais les pratiques religieuses et magiques auxquelles ont recours les migrants et leur famille avant, pendant et après le voyage révèlent la complexité de la construction des

\footnotetext{
${ }^{3}$ Même si le but de l'étude n'est pas ici d'établir une typologie des différentes pathologies liées aux migrations.
} 
discours associant (ou non) migration et santé mentale.

D’un point de vue méthodologique, selon Véronique Petit, travailler au Sénégal sur la santé mentale a été facilité par l'histoire de la naissance de la psychiatrie au Sénégal. Importés à l'époque de la colonisation, les services psychiatriques se mettent en place dans la première moitié du $\mathrm{XX}^{\text {ème }}$ siècle. C'est notamment sous l'influence d'Henri Collomb que se développe une approche culturelle de la psychiatrie visant à comprendre le fonctionnement socio-anthropologique de la société afin de faciliter la compréhension et le traitement des pathologies mentales. Cette tradition ethnopsychiatrique sénégalaise a largement facilité l'intégration de l'équipe de chercheurs de Miprimo dans les hôpitaux pour les enquêtes. Celles-ci se sont déroulées dans trois établissements: le Centre Hospitalier Universitaire (CHU) de Fann à Dakar, dont le service psychiatrique accueille plutôt des patients privilégiés, et qui représente la vitrine sénégalaise de la discipline; le service psychiatrique de l'Hôpital de Thiès, reconnu pour l'efficacité de ces traitements, il prend en charge des populations plus fragiles socialement et mentalement; l'Hôpital psychiatrique de Thiaroye, seul établissement du pays entièrement dédié à la psychiatrie et chargé d'une histoire lourde liée à l'enfermement d'opposants politiques, est aujourd'hui associé aux départs en pirogue des adolescents vers les îles Canaries. Ces trois services psychiatriques sont parmi les principaux du pays avec ceux de Saint-Louis et de Ziguinchor (envisagés comme futurs terrains pour compléter l'étude). Leur répartition sur le territoire est assez inégale et se concentre essentiellement sur la façade atlantique.

Le travail d'enquête s'est principalement articulé autour du recueil des récits de malades, du personnel, des accompagnants et des tradipraticiens et de séances d'observation dans les services psychiatriques. Les médecins et les malades ont accueilli cette enquête positivement, les malades, s'ennuyant, allant parfois jusqu'à solliciter eux-mêmes les entretiens avec les chercheurs. Même en considérant a priori la parole du malade comme légitime, Véronique Petit relève la difficulté des chercheurs à se confronter quotidiennement à la réalité des pathologies. Du fait de la variété des types et des degrés de pathologies, les entretiens avec les malades ont été extrêmement variés, mais cela a permis de nourrir le processus de déconstruction de la folie mené par les trois chercheurs sur ce terrain. Ce travail sur les récits oraux a été complété par des données issus de dossiers médicaux des patients. $\mathrm{Si}$ l'accès à ces informations - rendu possible par les autorisations des médecins - a posé aux chercheurs un problème déontologique par rapport aux questions relatives au secret médical, il a néanmoins permis de reconstituer les itinéraires migratoires et thérapeutiques des patients, permettant ainsi de s'intéresser plus particulièrement aux patients migrants. Le séjour au $\mathrm{CHU}$ de Fann à Dakar a également permis d'enrichir le corpus de documents d'archives tels que des journaux intimes, des lettres, des pièces de théâtres et d'autres matériaux thérapeutiques produits par les malades permettant de donner une vision plus positive de la folie, notamment en l'associant à la créativité. Des récits d'auteurs (Mariam Gueye et Abasse Ndione notamment) viennent compléter des entretiens avec des artistes qui s'intéressent au sujet de la folie et des journalistes dont les discours semblent être de plus en plus marqués par le vocabulaire de la psychiatrie.

Au travail de terrain fait suite un long travail d'analyse des récits. Certaines perspectives se dessinent pour la suite, notamment celle d'une étude des liens entre politiques migratoires et politique de santé, en s'intéressant à la façon dont les malades et leurs familles sont l'objet d'une attention particulière de la part du ministère de la Santé. D’un point de vue méthodologique, 
Véronique Petit exprime le désir de sortir du carcan hospitalier pour aller enquêter dans les familles, mais le projet pose pour l'instant la question de l'accès aux malades dans les familles en évitant les problèmes de stigmatisation. Ses questionnements s'élargissent également au devenir des migrants et au ratio sexuel des malades très déséquilibré en faveur des hommes. D'autres questions émergent quant aux conséquences sur la santé mentale de la famille (femmes, enfants) des migrants liées à l'absence d'un de ses membres.

Dans les discussions suivant la présentation du travail de Véronique Petit, les chercheurs présents au séminaire relèvent le grand intérêt méthodologique de la communication. L'originalité de la méthode de juxtaposition des différents types de récits retient également l'intérêt de tous. L'effort réflexif dans la restitution du travail de terrain a permis à Véronique Petit de présenter une version analytique de sa problématique de recherche en s'attachant toujours à déconstruire les représentations attachées aux questions de santé mentale. Cette séance de séminaire attise la curiosité quant à l'approfondissement de la réflexion méthodologique qui sera menée suite à l'analyse des résultats de ce travail de terrain.

Anne-Laure Counilh Doctorante en Géographie Migrinter UMR 7301 Université de Poitiers/CNRS alcounilh@gmail.com

\section{Bibliographie (indiquée par Véronique Petit)}

Amouzou Akoété (2011) From Malady to Self-Apprenhension and Nation Building: A Reading of Anthony Biakolo's Wonderful Child, in Kandji, M. (dir.) Health and Mental Issues in the literary Imagination, Dakar, Diaspora Academy Press, pp. 3-18.

Comaroff, Jean \& John (1987) The madman and the migrant. Work and Labor in the Historical Consciousness of a South African People, American Ethnologist, vol. 14, $\mathrm{n}^{\circ} 2$, pp. 191-209.

Foucault, Michel (1972) Histoire de la folie à l'âge classique, Paris, Gallimard, 642 p.

Gauchet, Marcel ; Swain, Gladys (2007) La pratique de l'esprit bumain. L'institution asilaire et la révolution démocratique, Paris, Gallimard, 519 p.

Kilroy-Marac, Kathleen (2009) The impossible inheritance: Memory and Postocolonial subjectivity at the Fann Hospital in Dakar, Senegal, New York, Columbia University, 238 p.

Ly, Mouhamed ; Petit, Véronique ; Pizzolato, Giulia (2014) La migration face à la santé mentale au Sénégal : récits, discours et imaginaires, in Canut, C.; Mazauric, C. (dir.) La migration prise aux mots : mise en récits et en images des migrations transafricaines, Paris, Éditions Le cavalier bleu.

Murat, Laure (2011) L'bomme qui se prenait pour Napoléon. Pour une bistoire politique de la folie, Paris, Gallimard, 416 p.

Thioub, Ibrahima (2003) L'enfermement carcéral : un instrument de gestions des marges urbaines au Sénégal XIXe-XXe siècles, Revue Canadienne des Etudes Africaines, vol. $37, n^{\circ} 2 / 3$, pp. 269-297. 